\title{
Phase Multistability of Synchronous Chaotic Oscillations
}

\author{
T.E. VADIVASOVA*, O.V. SOSNOVTSEVA, A.G. BALANOV and V.V. ASTAKHOV \\ Physics Department, Saratov State University, Astrokhanskaya str. 83, 410026, Saratov, Russia
}

(Received 15 April 1999)

\begin{abstract}
The paper describes the sequence of bifurcations leading to multistability of periodic and chaotic synchronous attractors for the coupled Rössler systems which individually demonstrate the Feigenbaum route to chaos. We investigate how a frequency mismatch affects this phenomenon. The role of a set of coexisting synchronous regimes in the transitions to and between different forms of synchronization is studied.
\end{abstract}

Keywords: Chaos, Synchronization, Multistability, Attractor

\section{INTRODUCTION}

Synchronization of chaotic systems has become a significant field of research in recent years. Different approaches to chaotic synchronization, its criteria and peculiarities of bifurcation mechanisms of desynchronization have been developed in [1-18]. It seems that the classical concept of synchronization related to the locking of the basic frequencies and instantaneous phases of oscillations or the suppression of the basic frequencies is acceptable for a certain class of chaotic systems those wherein the basic frequencies can easily be distinguished in the power spectrum [6-11]. The basic frequency corresponds to the period of the initial limit cycle which produces a chaotic attractor via a cascade of period-doubling bifurcations (Feigenbaum scenario). The transition from non- synchronous chaos to synchronous behavior may be diagnosed by different methods. Anishchenko et al. $[7,17]$ compare the results from numerical simulation as well as from full-scale experiments and diagnose the boundary of chaotic synchronization when the locking of the basic frequencies of chaotic oscillations takes place. In [9-11] this transition is determined from the behavior of the instantaneous mutual phases of oscillations. Finally, in $[16,18]$ the authors suggest a method based on calculation of the characteristic time of a system as the mean return time to a Poincare surface.

In the parameter space of a system there exist regions which correspond to the frequency and/or phase locking (Arnol'd tongues). Chaotic oscillations inside these regions are synchronous. For identical interacting chaotic systems complete (full) synchronization is observed for a strong enough

* Corresponding author. 
coupling strength $[2,3,5]$. It implies that timeseries of corresponding dynamical variables of subsystems coincide completely: $\quad \mathbf{x}_{\mathbf{1}}(t)=\mathbf{x}_{\mathbf{2}}(t)$. When a weak frequency mismatch is introduced lag synchronization appears, as a coincidence of the states of two systems: $\mathbf{x}_{\mathbf{1}}(t+\tau)=\mathbf{x}_{\mathbf{2}}(t)$ when shifted in time [11].

Multistability is another typical phenomenon observed in nonlinear dynamical systems. It is found commonly in many fields of science [19-23]. Many processes in nature are characterized by a large number of coexisting regimes for a fixed set of parameters but for different initial conditions. Due to a weak interaction multistability of periodic and chaotic attractors can be observed inside the synchronization region of mutually coupled units which individually follow the Feigenbaum perioddoubling route to chaos [17,24-27]. Let us call this type of multistability as the phase one because it is related to mutual synchronization with different phase relationships between oscillations, whose spectrum contains subharmonics of the basic frequency $\omega_{0}$. Possible synchronous regimes are increased in number when more subharmonics of the basic frequency can be distinguished in the power spectrum.

For initial periodic oscillations with period $T_{0}$ a phase difference $\phi_{0}$ between subsystems is equivalent a phase difference $\phi_{0}+2 \pi m, m=1,2, \ldots$ For oscillations with doubled period $2 T_{0}$ whose spectrum contains subharmonic $\omega_{0} / 2$, two different limit cycles in the phase space of interacting systems correspond to the phase differences $\phi_{0}$ and $\phi_{0}+2 \pi$. Thus, for two synchronized oscillators whose spectrum includes subharmonics $\omega_{0} / 2^{n}(n=1,2, \ldots)$ of the basic frequency, the phase difference between interacting units can attain $2^{n}$ different values, i.e. $\phi=\phi_{0}+2 \pi m, m=0,1,2, \ldots, 2^{n}-1$. Phase multistability also takes place for weak chaos, that demonstrates an $\mathrm{N}$-band structure. Hierarchy of multistability in identical interacting systems with dissipative coupling has been studied numerically and experimentally by Astakhov et al. [24-26]. It has been shown in $[17,27]$ that this type of multistability is structurally stable with respect to a weak mismatch between the basic frequencies. But the sequence of bifurcations is changed when the mismatch and the coupling strength are increased. Postnov et al. [27] described the nested structure of the phase synchronized region.

In this paper we study multistability phenomenon for two coupled Rössler systems. We show how a frequency mismatch affects a sequence of bifurcations leading to the growth of a number of coexisting attractors. We investigate different forms of synchronization which are related to the coexisting oscillatory regimes with different phase shifts.

\section{MODEL}

Synchronization is a universal nonlinear phenomenon, and its key features are typically independent of a model. As an example, we consider the system of coupled Rössler oscillators as described by Rosenblum et al. [9]:

$$
\begin{aligned}
\dot{x}_{1} & =-\omega_{1} y_{1}-z_{1}+\gamma\left(x_{2}-x_{1}\right), \\
\dot{y}_{1} & =\omega_{1} x_{1}+\alpha y_{1}, \\
\dot{z}_{1} & =\beta+z_{1}\left(x_{1}-\mu\right), \\
\dot{x}_{2} & =-\omega_{2} y_{2}-z_{2}+\gamma\left(x_{1}-x_{2}\right), \\
\dot{y}_{2} & =\omega_{2} x_{2}+\alpha y_{2}, \\
\dot{z} & =\beta+z_{2}\left(x_{2}-\mu\right),
\end{aligned}
$$

where the parameters $\alpha, \beta$ and $\mu$ govern the dynamics of each subsystem. $\gamma$ is the coupling parameter, $\omega_{1}=\Omega+\Delta$ and $\omega_{2}=\Omega-\Delta$ are the natural frequencies, and $\Delta$ is the mismatch between these frequencies. Throughout the paper we keep $\alpha=$ $0.15, \beta=0.2, \Omega=1.0$ and $\gamma=0.02$. The equations in (1) serve as a good model for real systems demonstrating period-doubling route to chaos, i.e. for electronic circuits [7,8], chemical [28] and biological [29] systems. Because synchronization between two systems involves phase relations the notion of the phase for chaotic oscillators and several methods to characterize the phase dynamics were intensively discussed $[9,10,11]$. To uniquely introduce an instantaneous amplitude and phase 
of a chaotic signal one can use the following representation:

$$
\begin{aligned}
x_{i}(t) & =A_{i}(t) \cos \Phi_{i}(t), \\
\hat{x}_{i} & =A_{i}(t) \sin \Phi_{i}(t) .
\end{aligned}
$$

Here, $A(t)$ and $\Phi(t)$ are the instantaneous amplitude and phase, respectively; $\hat{x}(t)=\hat{H} x(t)$ denotes Hilbert transformation [30]. This approach to the investigation of chaotic dynamics was firstly introduced by Rosenblum et al. [9]. In the case when the dynamical variables $x(t)$ and $y(t)$ are connected in a linear way (as for the Rössler system, for example) it is easy to apply the following substitution:

$$
\begin{aligned}
& x_{i}(t)=A_{i}(t) \cos \Phi_{i}(t), \\
& y_{i}(t)=A_{i}(t) \sin \Phi_{i}(t) .
\end{aligned}
$$

Here, $A(t)$ and $\Phi(t)$ are polar coordinates of the point $(x(t), y(t))$ in the $X Y$ plane. When phase locking of chaotic oscillations occurs the phase difference $\Phi_{1}-\Phi_{2}$ is bounded while outside the synchronization region it is an increasing or decreasing function of time $[9,10]$. Phase locking is related to the locking of basic frequencies in the power spectrum of chaotic oscillations.

\section{DYNAMICS OF TWO COUPLED IDENTICAL SYSTEMS}

In this section we study the dynamics of Eq. (1) as the parameter $\mu$ is varied in the case of completely identical partial oscillators (i.e., $\Delta=0$ ). As $\mu$ is increased, a sequence of bifurcations leading from the initial cycle of period $T_{0}$ located in the invariant symmetric subspace $U\left(x_{1}=x_{2}, y_{1}=y_{2}, z_{1}=z_{2}\right)$ to a set of multistable states is indicated. Before bifurcating to chaos a number of limit cycles coexisting in the phase space is increased. Let us denote the cycle with the period $2^{n} T_{0}$ and the phase shift $\phi=2 \pi m$ by the symbol $2^{n} C^{m}(n=0,1,2, \ldots$; $m=0,1,2, \ldots)$. A chaotic attractor with $2^{n}$ bands arising from the cycle with the phase shift $\phi=2 \pi m$ is labeled as $2^{n} C A^{m}$. To illustrate the oscillatory regimes of the system and transitions between them we show schematically in Fig. 1 the evolution of periodic and chaotic regimes when parameter $\mu$ is increased while the coupling strength $\gamma$ is fixed at 0.02 . Note that the branch $\mathbf{A}$ corresponds to "in-phase" oscillations (i.e., the phase shift between the oscillations is equal to zero and trajectories lay in $U$ ) while the branches $\mathbf{B}, \mathbf{C}$ and $\mathbf{D}$ illustrate the "out-of-phase" regimes originated from $2 C^{1}$, $4 C^{2}$ and $8 C^{4}$, respectively.

As $\mu$ increases, the "in-phase" limit cycle $C^{0}$ undergoes a period-doubling bifurcation. A cycle $\left(2 C^{0}\right)$ of twice the period emerges smoothly. The cycle $C^{0}$ which becomes saddle continues to exist and undergoes another period-doubling bifurcation. As a result of this bifurcation a saddle cycle $2 C^{1}$ of twice the period is born. This cycle is not located any longer in the symmetric subspace $U$ but it is self-symmetric with respect to the invariant manifold $U$ (i.e., $x_{1}=-x_{2}, y_{1}=-y_{2}$, $z_{1}=-z_{2}$ ). It becomes stable via inverse subcritical pitchfork bifurcation as $\mu$ is further increased. In the same manner, each "in-phase" limit cycle $2^{n} C^{0}$ gives rise the corresponding branch of "out-of-phase" regimes. For the above "out-ofphase" cycles the replacement of the next perioddoubling bifurcation by torus birth bifurcation takes place. The torus birth bifurcation leads to quasiperiodicity, frequency locking and emergence of new "out-of-phase" families of attractors which follow the period-doubling route to chaos. Beyond the threshold, a set of chaotic attractors coexists. As $\mu$ increases, there are the merging bifurcations where the number of bands in the attractor is halved. Besides this, a crises of chaotic limit sets leading to the merging of attractors of different branches take place. Finally, the only one-band global chaotic attractor $C A^{\Sigma}$ including the chaotic sets of all branches emerges in the phase space of the system.

A phase shift $2 \pi m$ between the oscillations of subsystems that defines the corresponding branch of regimes cannot be found using instantaneous phases $\Phi_{1,2}$ from (2) or (3). The instantaneous phases and their difference are determined with 


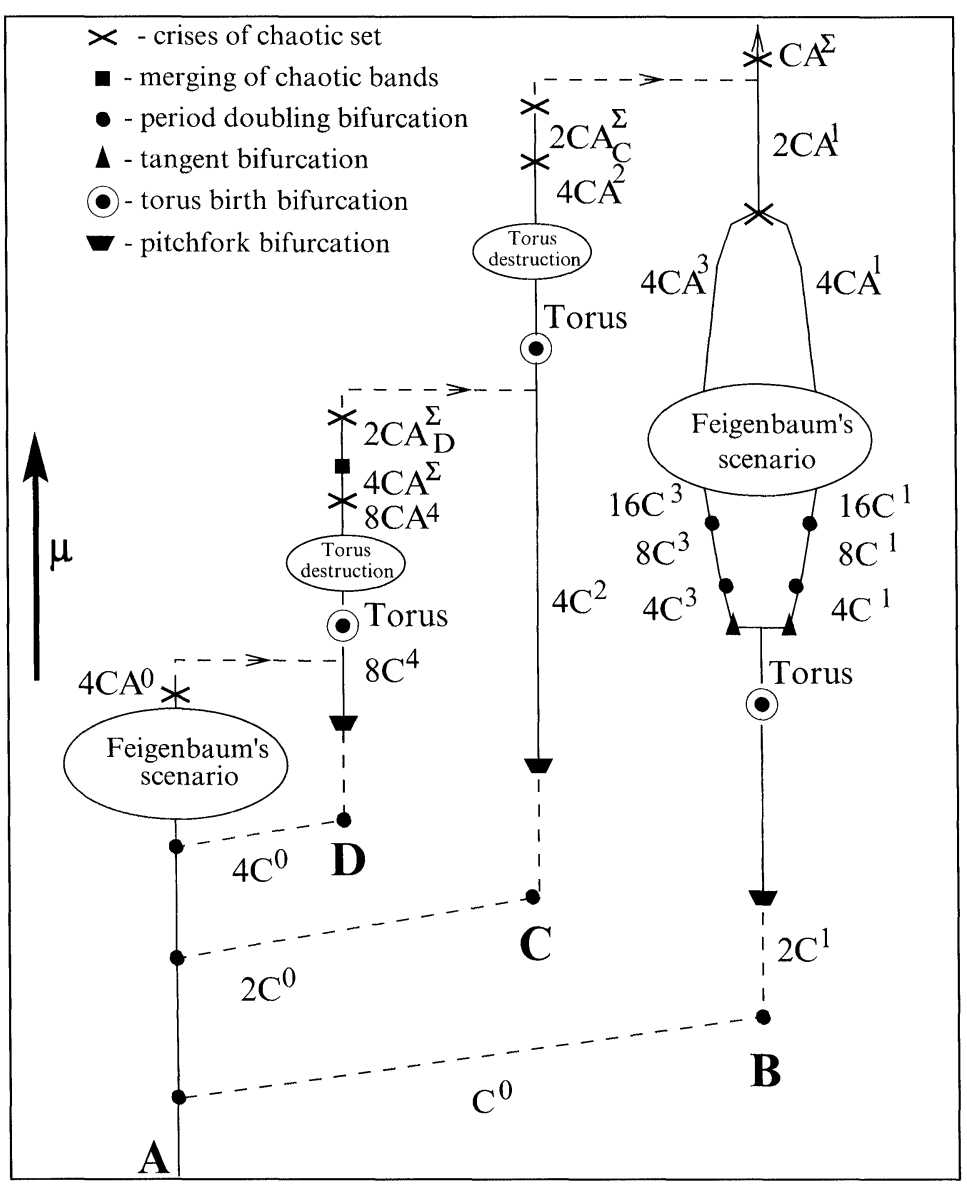

FIGURE 1 Evolution of oscillatory regimes for the identical coupled Rössler systems. The solid and dashed lines correspond to bifurcational transitions of attractive and saddle solutions, respectively.

the accuracy of $\pm 2 \pi k, k=1,2, \ldots$ Therefore, the difference of instantaneous phases for the attractors of all branches is limited within the range $[-\pi, \pi]$ if its initial value is chosen in this interval. To find a phase shift $2 \pi m$ it is necessary to determine a shift value in time (when $\Delta=0$ ) and re-write it in polar coordinates. Taking into account that "out-of-phase" regimes are located out of the symmetric subspace $U$, we may introduce a time shift when states of subsystems coincide but lagged with respect to each other. It can be calculated via the global minimum of a similarity function $S$ as described in [11]:

$$
S^{2}(\tau)=\frac{\left\langle\left(x_{2}(t+\tau)-x_{1}(t)\right)^{2}\right\rangle}{\sqrt{\left(\left\langle x_{1}^{2}(t)\right\rangle\left\langle x_{2}^{2}(t)\right\rangle\right)}},
$$

where the angular brackets assume the averaging over time.

Let us consider the evolution of chaotic attractors in detail. Note, that there are three types of crises labeled in Fig. 1 as "cross": a transformation of a chaotic attractor into a chaotic saddle, a merging of chaotic attractors of the same branch, and merging of a chaotic attractor of one branch with a chaotic attractor of another branch. As $\mu$ is increased, at the branch $\mathbf{A}$ a chaotic attractor which appears via a period-doubling cascade of "inphase" regimes and which is located in the subspace $U$ bifurcates in a chaotic saddle. At this moment, the second positive Lyapunov exponent of chaotic trajectories in $U$ is indicated. It corresponds to the additional unstable direction which is transverse 
to $U$. This transition leads to the loss of complete synchronization. The mechanism of similar transitions was studied in [31-35]. When an initial point on $U$ is slightly perturbed and after a long transient time, a phase trajectory tends to stay at a stable cycle $8 C^{4}$ of the branch $\mathbf{D}$. When $\mu$ is further increased, a sequence of bifurcations of this cycle leads to chaotic attractor $8 C A^{4}$ (Fig. 2(a)) which at $\mu \approx 6.036$ undergoes a crisis with a chaotic saddle of the branch $\mathbf{A}$ as well as a band merging As a result, the branches $\mathbf{A}$ and $\mathbf{D}$ merge together with appearance of a chaotic attractor $4 C A_{D}^{\Sigma}$ (Fig. 2(b)). This merging crisis is accompanied by "on-off" intermittency. Then $2 C A_{D}^{\Sigma}$ appears from $4 C A_{D}^{\Sigma}$. At $\mu \approx 6.06$ a chaotic attractor $2 C A_{D}^{\Sigma}$ becomes a saddle. After this transition, phase trajectories switch to the stable cycle $4 C^{2}$ which belongs to the branch C. Chaotic attractor $4 C A^{2}$ (Fig. 2(c)) appears from $4 C^{2}$ via a sequence of bifurcations. At $\mu \approx 6.35$, the merging crisis of a chaotic attractor $4 C A^{2}$ and a saddle of the branch $\mathbf{D}$ occurs and a new chaotic attractor $2 C A_{C}^{\Sigma}$ emerges (Fig. 2(d)). At $\mu \approx$ 6.44 , this attractor becomes a saddle but a phase trajectory jumps to a chaotic attractor $2 C A^{1}$ (Fig. 2(e)) of the branch B. Then at $\mu \approx 6.70$, the merging of the chaotic attractor $C A^{1}$ with a saddle of the branch $\mathbf{C}$ occurs. Thus, a sequence of crises ends as the one chaotic attractor $C A^{\Sigma}$ (Fig. 2(f)) which involves chaotic trajectories from all branches.

It has been found that the behavior of the phase difference calculated from (3) is different for a variety of chaotic attractors inside the synchronization region. For chaotic attractor located in the symmetric subspace ( $4 C A^{0}$, for instance), it is constant in time $\left(\delta \Phi(t)=\Phi_{1}(t)-\Phi_{2}(t)=0\right)$. For "out-ofphase" attractors it is not equal to zero and varied chaotically in time. Width of the distribution of phase differences $P(\delta \Phi)$ characterizes the "degree of antiphase" of an attractor. Figure 3 displays the distribution of phase differences for the chaotic attractors $4 C A_{D}^{\Sigma}, 2 C A_{C}^{\Sigma}$, and $C A^{\Sigma}$. It is clearly seen that the merging of chaotic sets from different families (branches) leads to the expansion of the distribution function. But note, that $\delta \Phi$ remains bounded in the interval $[-\pi, \pi]$ since the described chaotic attractors are synchronous.
The chaotic attractor $C A^{\Sigma}$ corresponds to the regime of hyperchaos. But the regime with two positive Lyapunov exponents appears before than $C A^{\Sigma}$ is formed. For example, the chaotic attractor $2 C A^{1}$ of the branch $\mathbf{B}$ which appears via a merging of $4 C A^{1}$ and $4 C A^{2}$ has two positive Lyapunov exponents. For the branches $\mathbf{C}$ and $\mathbf{D}$, the transition to hyperchaos is observed when a torus is destroyed.

\section{EFFECT OF FREQUENCY MISMATCH}

In this section we introduce a mismatch between the basic frequencies in the system of coupled oscillators and study the evolution of multistability and different forms of synchronization.

Figure 4(a) and (b) shows the bifurcation diagrams of the synchronization region for attractors from two branches $\mathbf{A}$ and $\mathbf{B}$, respectively. It has been found that a small frequency mismatch $(|\Delta| \leq 0.001)$ does not almost affect the evolution of different oscillatory regime which is observed in the case of vanishing mismatch. Note, that at $\Delta \neq 0$ the invariant subspace $U$ does not exist any longer and the relations of symmetry for limit sets are not satisfied. Therefore, pitchfork bifurcations of limit cycles are replaced by tangent bifurcations leading to the birth of saddle "out-ofphase" cycles [35].

When a frequency mismatch is increased ( $\Delta \geq 0.0015)$, the period-doubling bifurcations for cycles $2 C^{1}, 4 C^{2}, \ldots$ are observed instead of torus birth bifurcations (Fig. 4(b)). Moreover, types of merging crises of chaotic sets depend on the mismatch value. With a frequency mismatch, there is no merging of chaotic attractors $4 C A^{1}$ and $4 C A^{3}$ but attractor $4 C A^{3}$ becomes a saddle and then it merges with the attractor $4 C A^{1}$. Transition to hyperchaos occurs before this crisis.

Let us consider the effect of frequency mismatch in terms of synchronization. Chaotic attractor $4 C A^{0}$ of the branch $\mathbf{A}$, located in the symmetric subspace $U$ in the case of $\Delta=0$, does not belong to $U$ when $\Delta \neq 0$. Hence, complete synchronization is lost. However, for a weak frequency mismatch this 

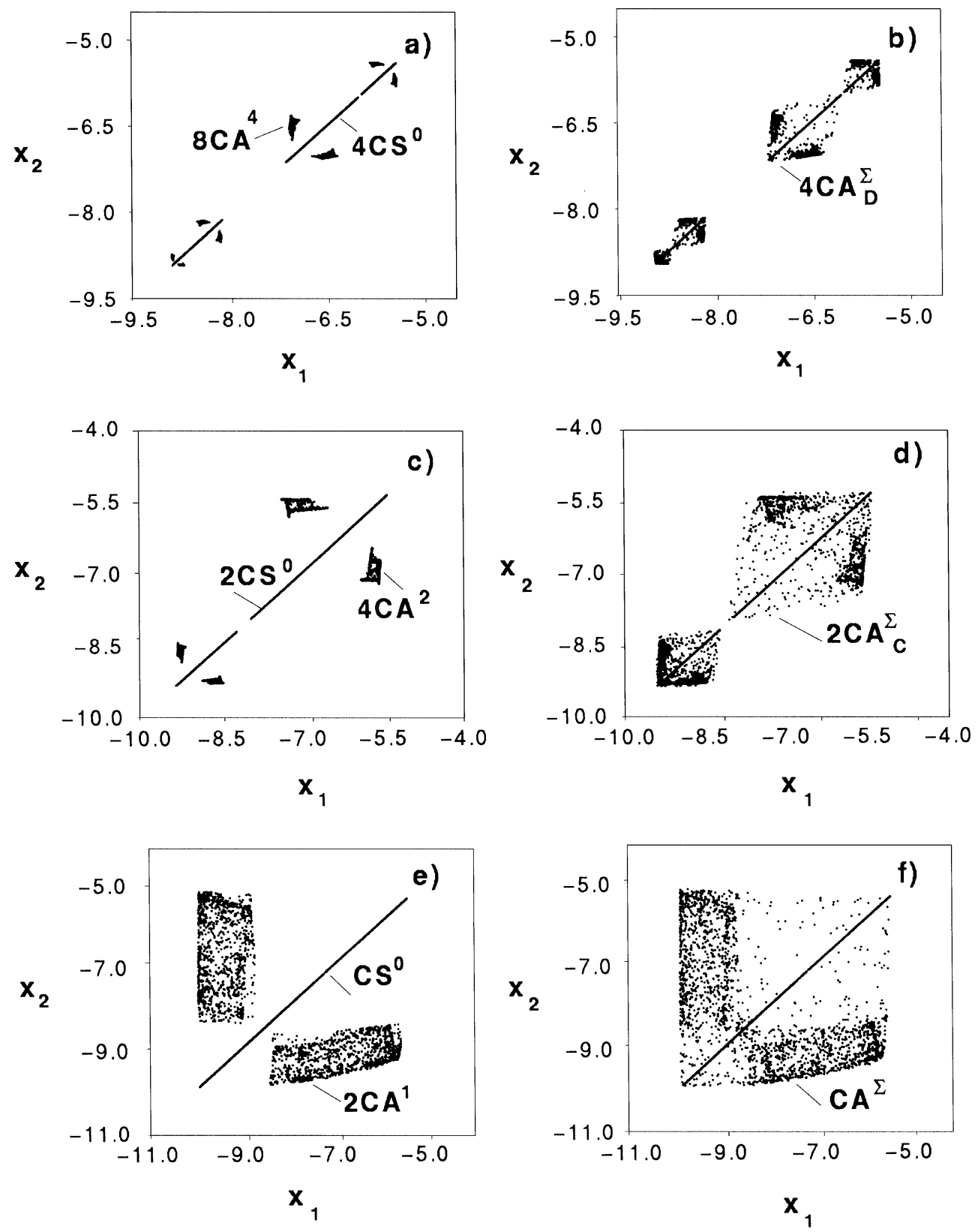

FIGURE 2 Projections of Poincaré sections of chaotic sets for the identical coupled Rössler systems. Secant plane is specified as $y_{1}=0$. Label "CS" is used to identify a saddle set.

regime remains topologically equivalent to the attractor in $U$. In this case referred to as lag synchronization [11], the oscillations of two systems coincide but shifted in time. For chaotic attractors of other families and attractors appearing via merging of chaotic sets from different branches full coherence cannot be achieved. They demonstrate only phase coherence properties. 

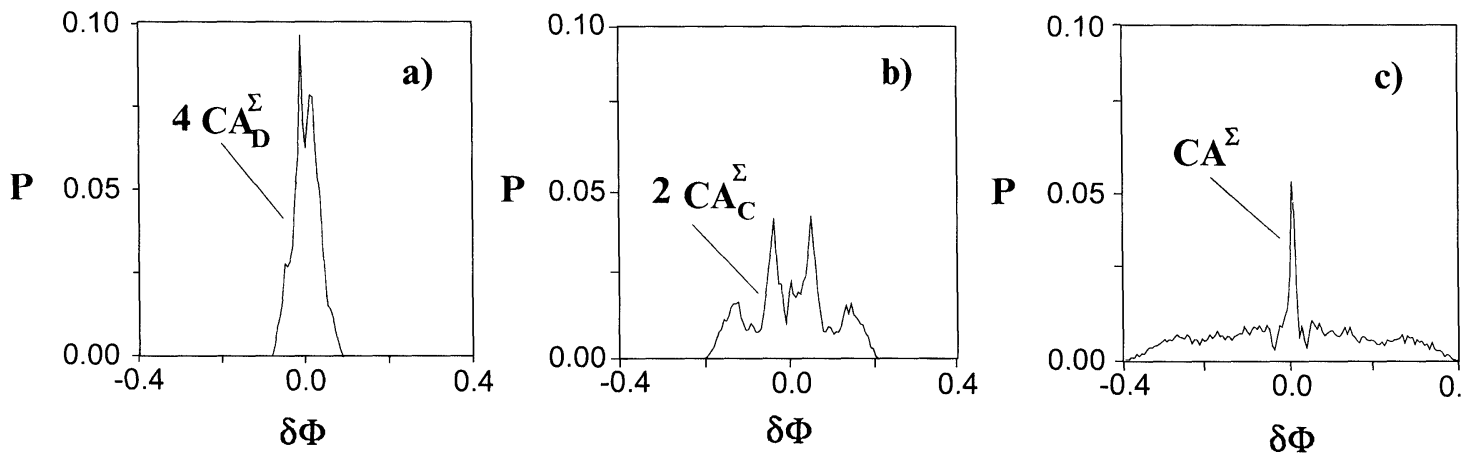

FIGURE 3 The distribution of phase difference for "out-of-phase" attractors: (a) $4 C A_{D}^{\Sigma}$ at $\mu=6.038$; (b) $2 C A_{C}^{\Sigma}$ at $\mu=6.36$; (c) $C A^{\Sigma}$ at $\mu=6.72$. Calculations were performed with the constant step $2 \pi / 100$.
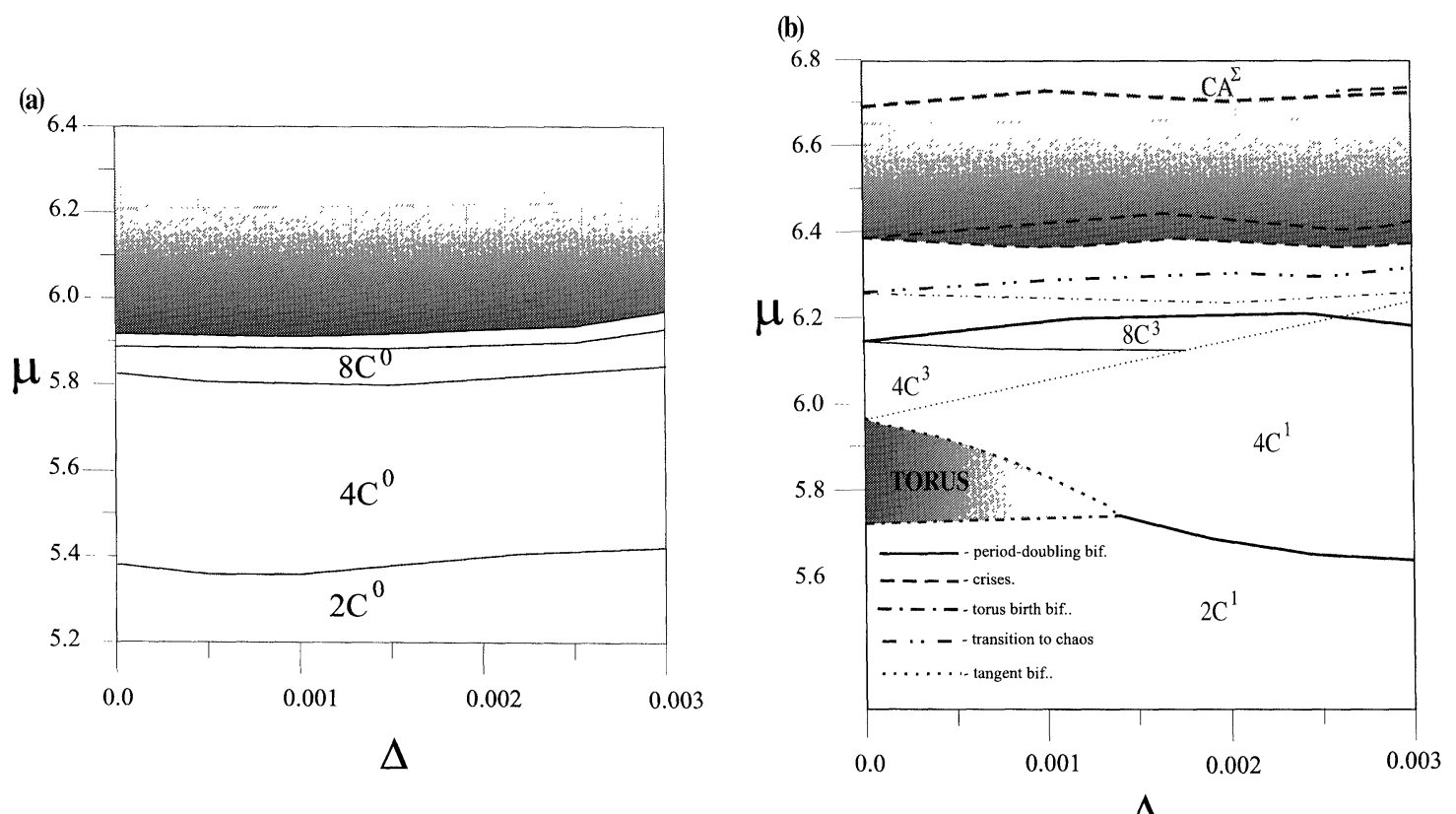

FIGURE 4 Bifurcation diagram on $(\Delta-\mu)$ parameter plane for the attractors of the branches $\mathbf{A}$ (a) and $\mathbf{B}$ (b). Curves of different width correspond to different families of attractors.

\section{PHASE TRANSITIONS NEAR THE BOUNDARY OF SYNCHRONIZATION REGION}

A bifurcational mechanism of the phenomena that take place at the boundary of chaotic phase synchronization is associated with the bifurcations of the saddle periodic orbits. Anishchenko et al. [7] have described this boundary as an accumulation of curves of tangent bifurcations of saddle cycles. Pikovsky et al. [14] suggest (for the model twodimensional map) that attractor-repeller collisions take place at the transition to chaotic synchronization, thus drawing an analogy with the tangent bifurcation of a limit cycle. Most recently [15], the transition to phase synchronization was considered as a boundary crisis mediated by unstable-unstable pair bifurcations on a branched manifold. We are 
interested in the transition between different coexisting regimes near the boundary of the phase synchronization region.

When a mismatch between the basic frequencies of interacting oscillators is introduced the regions of chaotic phase synchronization, similar to Arnol'd tongues for periodic oscillations, appear on the parameter plane. Hierarchy of multistability of synchronous regimes near the boundary (Fig. 5) differs from the case of $\Delta=0$. Taking into account the different sequence of bifurcations for periodic solutions that has been described in previous section, we focus on the peculiarities of the behavior of chaotic attractors. For a large mismatch, "outof-phase" attractors of the branches $\mathbf{B}$ and $\mathbf{C}$ become the saddles. When $\mu$ is increased, they merge with "in-phase" attractor of the branch $\mathbf{A}$.
Thus, attractor $4 C A_{A}^{\Sigma}$ appears via merging of an attractor $4 C A^{0}$ and a saddle of the branch $\mathbf{C}$. The band-merging crisis takes place and an attractor $2 C A_{A}^{\Sigma}$ arises. At this moment the transition to hyperchaos occurs. Then the merging crisis of $2 C A_{A}^{\Sigma}$ and a saddle of the branch $\mathbf{B}$ originated from attractor $4 C A^{1}$ leads to the single attractor $2 C A^{\Sigma}$ in the phase space of the system. Figure 6 shows the projections of Poincare sections of coexisting chaotic attractors $4 C A_{A}^{\Sigma}$ and $4 C A^{1}$ (Fig. 6(a)) and attractor $2 C A^{\Sigma}$ (Fig. 6(b)) which born as a result of merging of chaotic sets from all branches.

Figure 7 represents the bifurcation diagram of the synchronization region near the boundary. A nested structure of phase synchronized region for the attractors of the branches $\mathbf{A}$ and $\mathbf{B}$ is observed. With

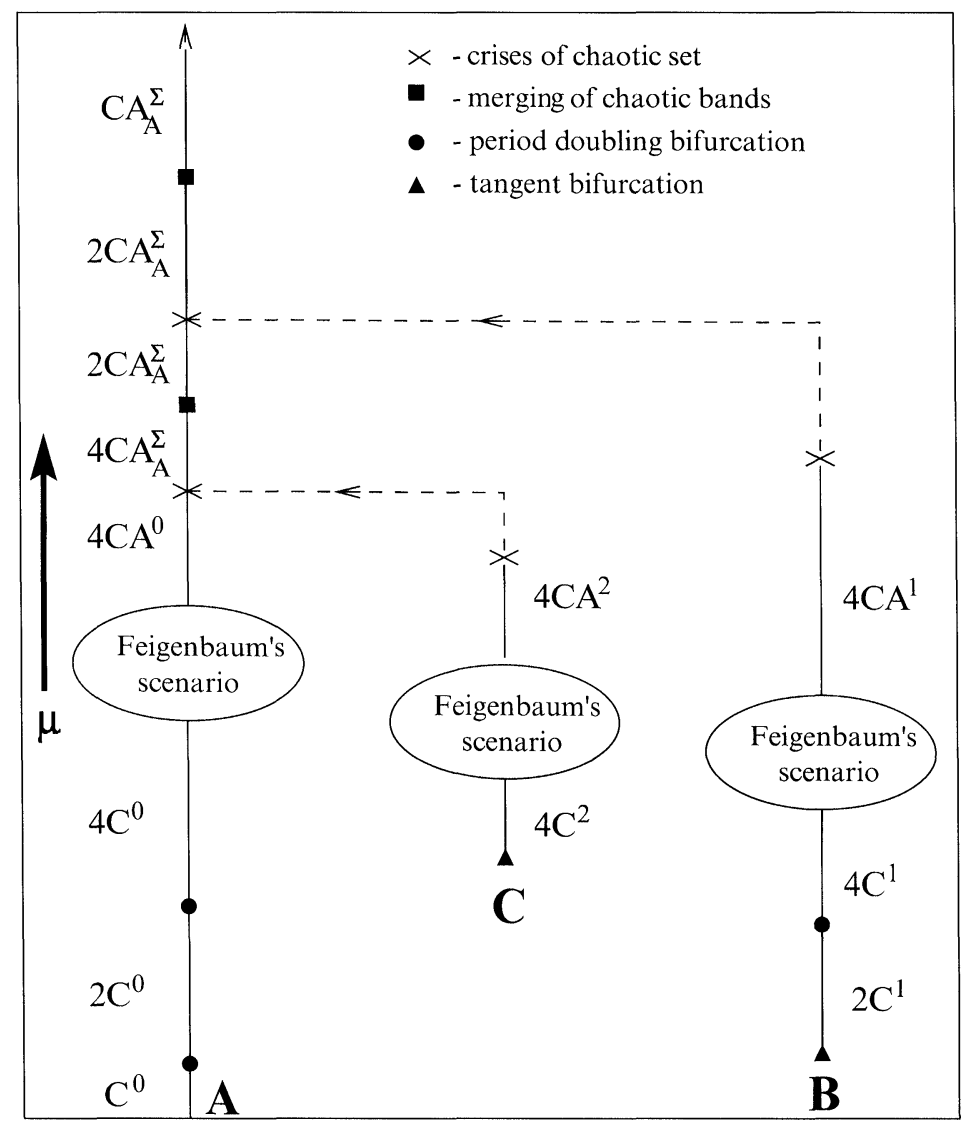

FIGURE 5 Evolution of oscillatory regimes when $\Delta=0.0093$. 

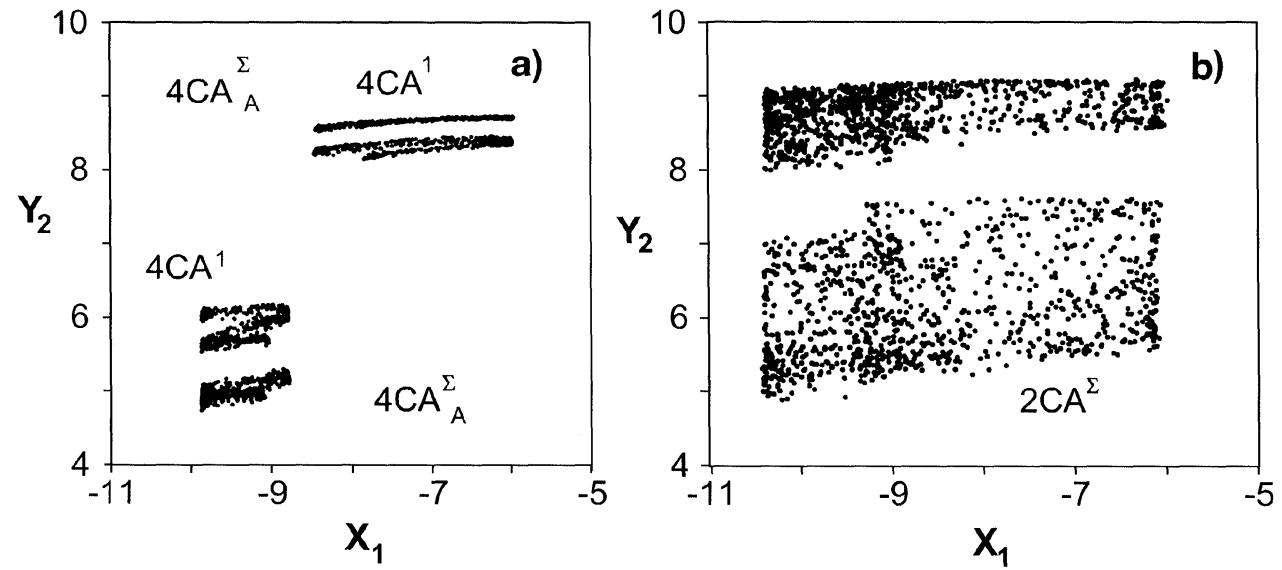

FIGURE 6 Projections of Poincaré sections of chaotic sets from the system (1) when $\Delta=0.0093$ at (a) $\mu=6.8$ and (b) $\mu=7.2$.

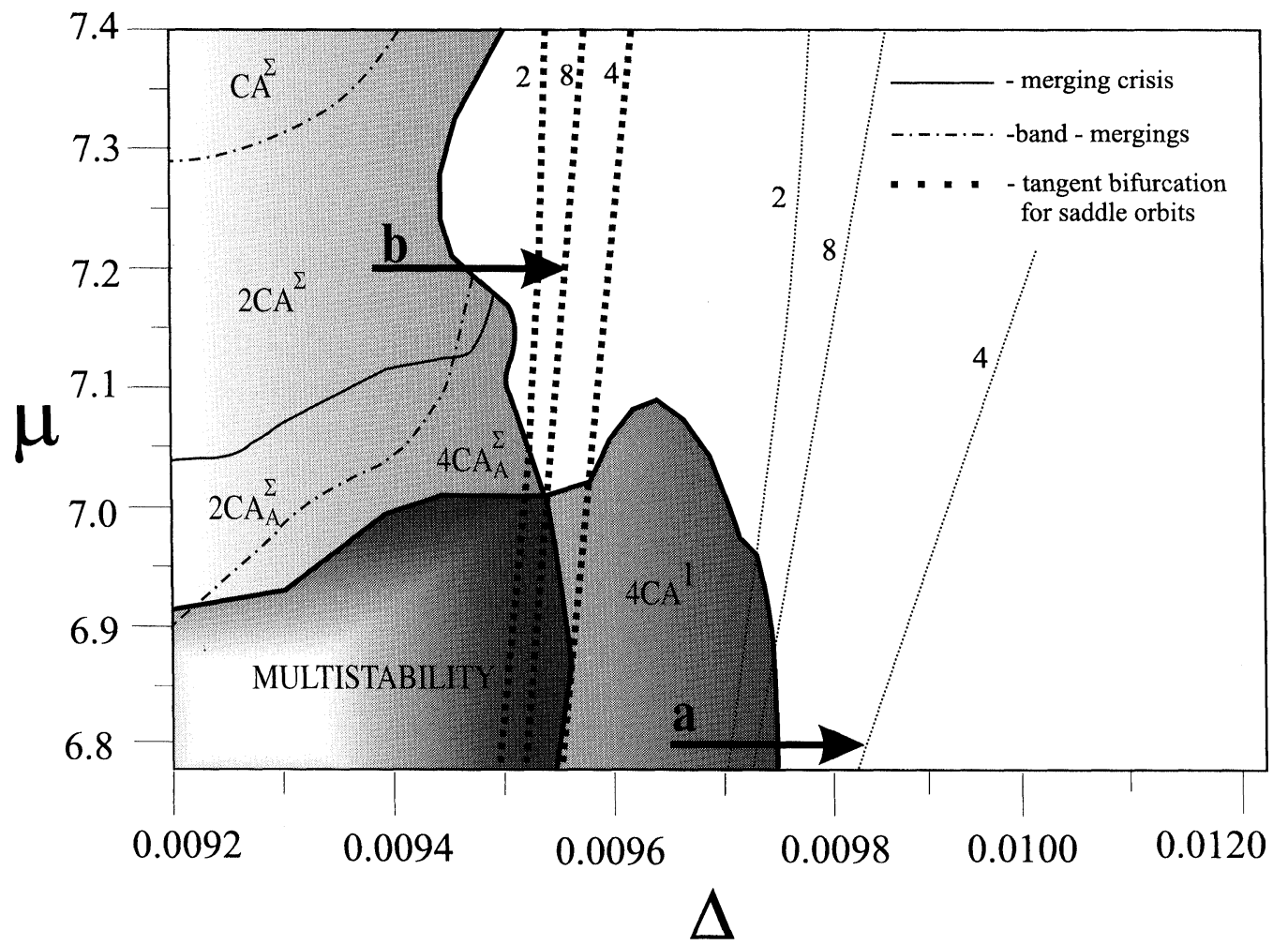

FIGURE 7 Bifurcational diagram near the boundary of the synchronization region. Dotted curves of different width correspond to the attractors of the branches $\mathbf{A}$ and $\mathbf{B}$. Numbers $\mathbf{2 , 4}$, and $\mathbf{8}$ denote the periods of saddle cycles.

this structure, the transition to non-synchronous behavior in the region of multistability (direction a in Fig. 7) is determined by the loss of stability for the most robust synchronous mode (the branch $\mathbf{B}$ in our case). Chaotic attractors of the branch $\mathbf{A}$ found to be structurally stable when $\mu$ is increased. Hence, above the region of multistability the transition from complex chaotic regimes, appearing 
after a set of merging crises, to non-synchronous dynamics (direction b) is observed.

The boundary of the synchronization region is diagnosed by the calculation of the spectrum of Lyapunov exponents and the diffusion coefficient of a phase difference. The later is described as follows:

$$
D(\Delta)=\lim _{t \rightarrow \infty} \frac{\delta \Phi(t)}{t} .
$$

Figure 8 displays these characteristics of synchronization along the direction a marked in Fig. 7 at $\mu=6.8$. It is clearly seen (Fig. 8(a)) that one of the negative Lyapunov exponents becomes equal to zero at the boundary of the synchronization region (a vertical dashed line). The same behavior has been observed for Rössler system with periodic forcing [16]. The coefficient of diffusion is vanishing inside the synchronization region but at the boundary it starts to grow (Fig. 8(b)). Similar calculations has been performed along the other direction b marked in Fig. 7. Figure 9(a) and (b) show that the behavior of Lyapunov exponents is not changed while the coefficient of diffusion is very sensitive to the transition to a non-synchronous regime.
Based on the results from $[7,14,15]$ where the bifurcation mechanism of phase synchronization is related to the bifurcations of saddle periodic orbits embedded in a chaotic attractor, we constructed the curves of tangent bifurcations of saddle cycles from the branches $\mathbf{A}$ and $\mathbf{B}$ (bold and thin dotted lines in Fig. 7, respectively). It is clearly seen that while multistability exists the curves tend to be located near the synchronization boundary of each branch of attractors. However, as soon as merging crises occur this approach becomes wrong. The question: what is the bifurcational transition from the merged synchronous regime which is characterized by two positive Lyapunov exponents, to non-synchronous behavior, is still open.

\section{SUMMARY OF THE RESULTS}

The phenomenon of phase multistability is the significant feature of phase synchronization in the systems with Feigenbaum route to chaos. Phase multistability determines the variety and complexity of bifurcation transitions inside the synchronization region and near its boundary. From the

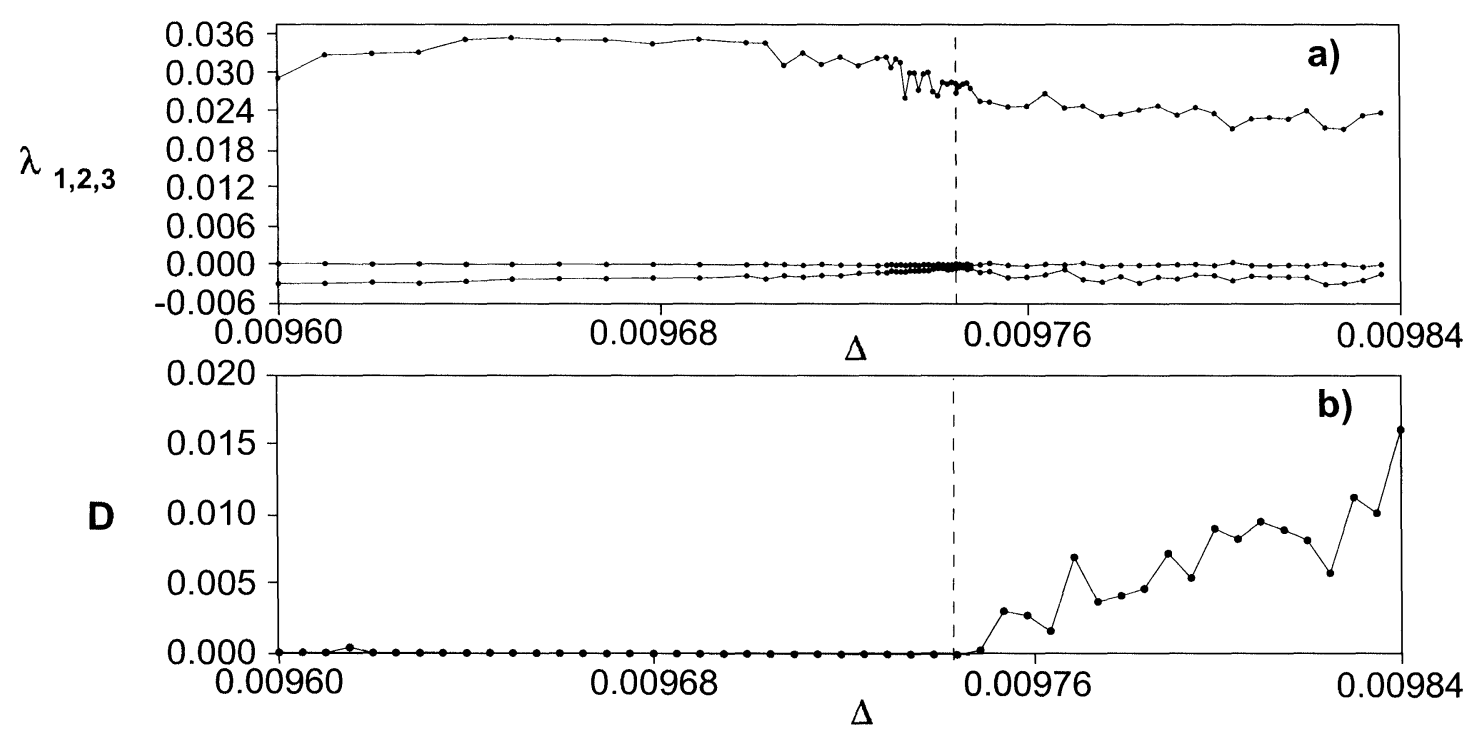

FIGURE 8 (a) The three largest Lyapunov exponents and (b) the coefficient of diffusion of phase difference as functions of $\Delta$ at $\mu=6.8$ (direction a in Fig. 7). 

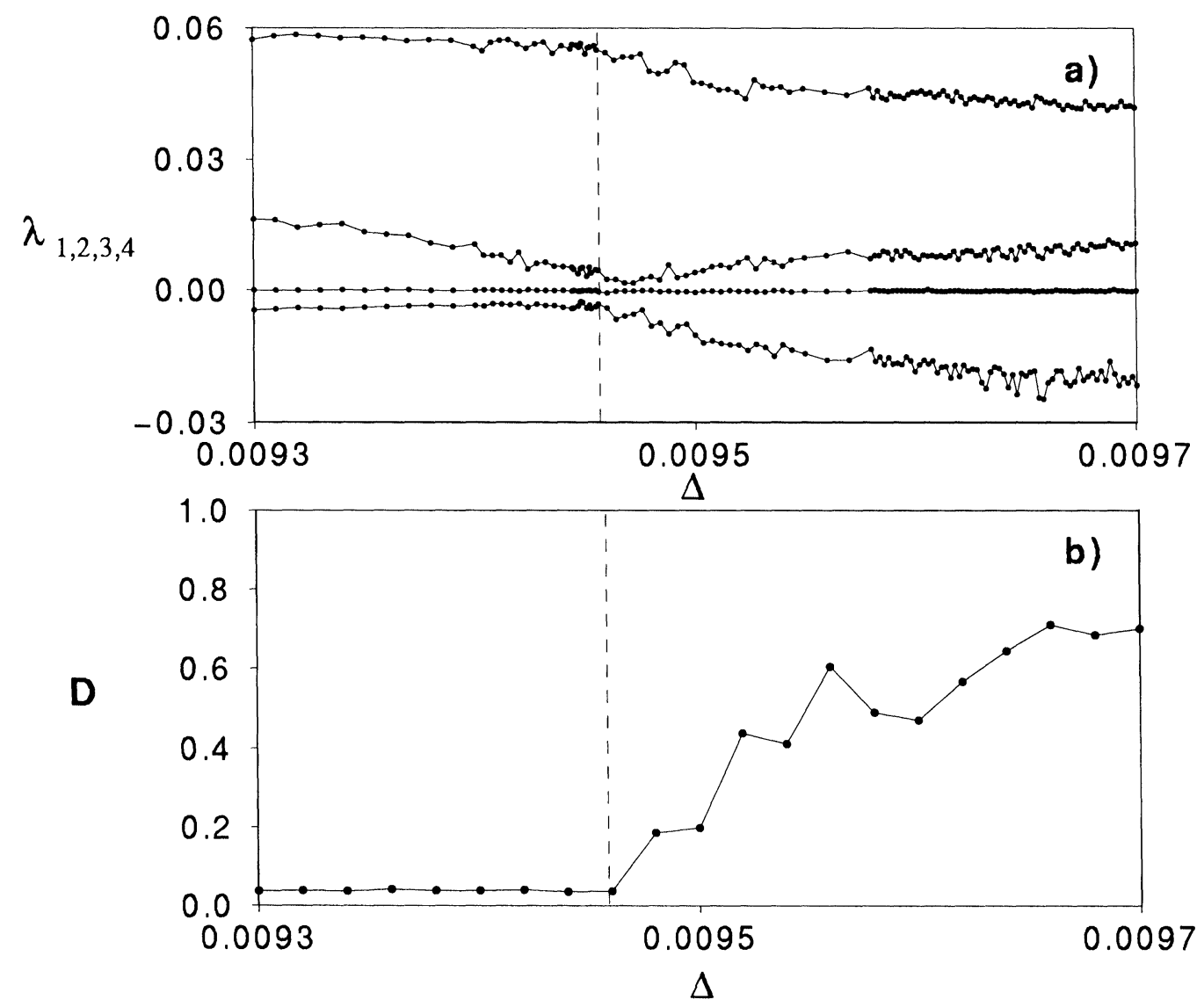

FIGURE 9 (a) The four largest Lyapunov exponents and (b) the coefficient of diffusion of phase difference as functions of $\Delta$ at $\mu=7.2$ (direction b in Fig. 7).

results presented above we can draw the following conclusions.

Two mutually coupled identical oscillators individually following the period-doubling route to chaos demonstrate the phenomenon of phase multistability, i.e. simultaneous existence of different periodic and chaotic attractors in the phase space of the system. This phenomenon is related to the possibility of various phase shifts between the oscillations of the partial systems. The number of coexisting regimes on the threshold of chaos inside the synchronization region for a weak coupling may be huge. As the control parameter is increased within the chaotic region a sequence of crises takes place: band-merging bifurcation that reduces the number of possible synchronous regimes by two; a merging of the different chaotic attractive and saddle sets. These transitions lead to the formation of a single hyperchaotic regime in the phase space of the system. In coupled Rössler oscillators the bifurcation scenario leading to multistability is similar to that observed for the other models with Feigenbaum route to choas and its main features are independent on the particular properties of a model.

A set of coexisting attractors of a system is structurally stable with respect to a mismatch between the basic frequencies. But the sequence of bifurcations can be different because of the symmetry relations are destroyed.

A nested structure of phase synchronized regions for different coexisting attractors is observed. We 
demonstrate that a regime of complete synchronization for the "in-phase" attractor is transferred into lag regime when a frequency mismatch is introduced. At the same time, the "out-of-phase" attractors also exist. They correspond to the regimes which are not lag synchronized but remain to be phase synchronized.

In the region of phase multistability, the transition to non-synchronous behavior is determined by the loss of stability for the most stable synchronous mode. The boundary of synchronization is easily diagnosed by phase portraits, the behavior of Lyapunov exponent, etc. The boundary structure is related to the bifurcations of saddle periodic orbits embedded in a synchronized chaotic set. However, in the region of hyperchaos the mechanism of the loss of synchronization is not studied in detail and needs further investigations.

\section{Acknowledgments}

This work is supported in part by RFFI Grant (9902-17732) and Grant (98-02-16531). Authors are grateful to D.E. Postnov and V.S. Anishchenko for helpful discussion.

\section{References}

[1] G. Dykman, P. Landa and Y. Neimark (1992). Synchronising of chaotic oscillations by external force. Chaos, Soliton. Fractal., 1(4), 339-353.

[2] H. Fujisaka and Y. Yamada (1983). Stability theory of synchronized motions in coupled oscillators systems. Progr. Theor. Phys., 69, 32.

[3] V.S. Afraimovich, N.N. Verichev and M.I. Rabinovich (1986). Stochastic synchronization of oscillations in dissipative systems. Radiophys. Quantum Electronics, 29(9), 795803.

[4] A.P. Volokovsky and N.F. Rulkov (1989). Experimental investigations of bifurcations on the onset of stochastical synchronization. Pis'ma v GTF, 15(7), 5-10 (in Russian).

[5] L. Pecora and T. Carroll (1990). Synchronization in chaotic systems. Phys. Rev. Lett., 64(8), 821-824.

[6] V.S. Anishchenko and D.E. Postnov (1988). Effect of the locking of the basic frequency for chaotic self-oscillations. Synchronization of strange attractors. Pis'ma v GTF, 14(6), 569-573 (in Russian).

[7] V.S. Anishchenko, T.E. Vadivasova, D.E. Postnov and M.A. Safonova (1992). Synchronization of chaos. Int. J. Bifurcation Chaos, 2(3), 633-644.

[8] J.F. Heagy, T.L. Carroll and L.M. Pecora (1994). Synchronous chaos in coupled oscillator systems. Phys. Rev. E, 50, 1874-1885.
[9] M. Rosenblum, A. Pikovsky and J. Kurths (1996). Phase synchronization of chaotic oscillators. Phys. Rev. Lett., 76, $1804-1807$.

[10] A.S. Pikovsky, M.G. Rosenblum, G.V. Osipov and J. Kurths (1997). Phase synchronization of chaotic oscillators by external driving. Physica $D, \mathbf{1 0 4}, 219-238$.

[11] M.G. Rosenblum, A.S. Pikovsky and J. Kurths (1997). From phase to lag synchronization in coupled chaotic oscillators. Phys. Rev. Lett., 78, 4193-4196.

[12] N.F. Rulkov, M.M. Sushchik, L.S. Tsimring and H.D.I. Abarbanel (1995). Generalized synchronization of chaos in unidirectorally coupled chaotic systems. Phys. Rev. E., 51, 980-995.

[13] L. Kocarev and U. Parlitz (1996). Generalized synchronization, predictability, and equivalence of unidirectionally coupled dynamical systems. Phys. Rev. Lett., 76(11) 18161819.

[14] A. Pikovsky, G. Osipov, M. Rosenblum, M. Zaks and J. Kurths (1997). Attractor-repeller collision and eyelet intermittency at the transition to phase synchronization. Phys. Rev. Lett., 79, 47-50.

[15] E. Rosa, E. Ott and M.H. Hess (1998). Transition to phase synchronization of chaos. Phys. Rev. Lett., 80(8), $1642-1645$

[16] T.E. Vadivasova, A.G. Balanov, O.V. Sosnovtseva, D.E. Postnov and E. Mosekilde (1999). Synchronization in driven chaotic systems: Diagnostics and bifurcations. Phys. Lett. A, 253, 66-74.

[17] V.S. Anishchenko, V.V. Astakhov, T.E. Vadivasova, O.V. Sosnovtseva, C.W. Wu and L.O. Chua (1995) Dynamics of two coupled Chua's circuits. Int. J. Bifurcation Chaos, 5(6), 1677-1699

[18] V.S. Anishchenko, A.N. Silchenko and I.A. Khovanov (1998). Synchronization of switching processes in coupled Lorenz systems. Phys. Rev. E, 57, 316-322.

[19] P.M. Battelino, C. Grebogi, E. Ott and J.A. Yorke (1988). Multiple coexisting attractors, basin of boundaries and basic sets. Physica D, 32, 296-305.

[20] S. Kim, S.H. Park and C.S. Ryu (1997). Noise-enchanced multistability in coupled oscillator systems. Phys. Rev. Lett., 78, 1616-1619.

[21] M. Brambilla, L.A. Lugiato and V. Penna (1991). Variational principle for pattern selection, spatial multistability and laser hydrodynamics. Phys. Rev. A, 43, 5114-5120.

[22] J. Foss, A. Longin, B. Mensour and J. Milton (1996). Noise, multistability and delayed recurrent loops. Phys. Rev. Lett., 76, 708-711.

[23] U. Feudel and C. Grebogi (1997). Multistability and the control of complexity. Chaos, 7(4), 597-604.

[24] V.V. Astakhov, B.P. Bezruchko, V.I. Ponomarenko and Ye.P. Seleznev (1988). Quasiuniformal stochastic motions and their destruction in the system of coupled nonlinear oscillators. Izv. Vuzov. Radiofizika, 31, 627-630 (in Russian).

[25] V.V. Astakhov, B.P. Bezruchko, Ju.V. Guljaev and Ye.P. Seleznev (1988). Multistable regimes of dissipatively-coupled Feigenbaum systems. Pis'ma v GTF., 15(3), 60-64 (in Russian).

[26] V.V. Astakhov, B.P. Bezruchko, E.N. Erastova and E.P. Seleznev (1990). Oscillation types and their evolution in dissipatively coupled Feigenbaum systems. Sov. Tech. Phys., 35, 1122-1129.

[27] D.E. Postnov, T.E. Vadivasova, O.V. Sosnovtseva, A.G. Balanov, V.S. Anishchenko and E. Mosekilde (1999). Role of multistability in the transition to chaotic phase synchronization. Chaos, 9(1), 227-232. 
[28] A. Goryachev and R. Kapral (1996). Spiral waves in chaotic systems. Phys. Rev. Lett., 76, 1619-1622.

[29] E. Mosekilde (1996). Topics in Nonlinear Dynamics. Applications to Physics, Biology and Economics Systems. World Scientific, Singapore.

[30] P. Parter (1965). Modulation, Noise, and Spectral Analysis. McGraw-Hill, New York.

[31] A. Pikovsky and P. Grassberger (1991). Symmetry breaking bifurcation for coupled chaotic attractors. J. Phys. A: Math. Gen., 24, 4587-4597.

[32] P. Ashwin, J. Buescu and I. Stewart (1994). From attractor to chaotic saddle: Tale of transverse instability. Nonlinearity, 9, 703-737.
[33] M. Sushchik, N.F. Rulkov and D.I. Abarbanel (1997). Robustness and stability of synchronized chaos: An illustrative model. IEEE: Trans. Circuit. System., Fundamental Theory and Applications, 44(10), 867-873.

[34] V. Astakhov, A. Shabunin, T. Kapitaniak and V. Anishchenko (1997). Loss of chaos synchronization through the sequence of bifurcations of saddle periodic orbits. Phys. Rev. Lett., 79(6), 1014-1017.

[35] V. Astakhov, M. Hasler, T. Kapitaniak, A. Shabunin and V. Anishchenko (1998). The effect of parameter mismatch on the mechanism of chaos synchronization loss in coupled systems. Phys. Rev. E, 58(5), 5620-5628. 


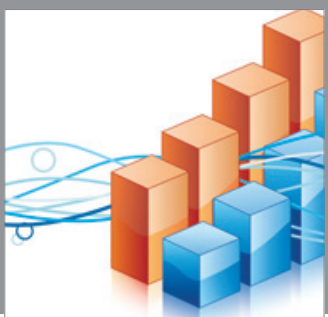

Advances in

Operations Research

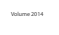

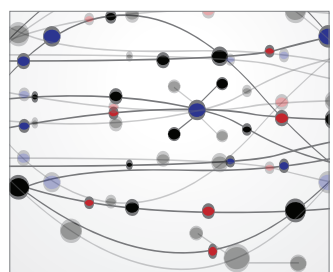

\section{The Scientific} World Journal
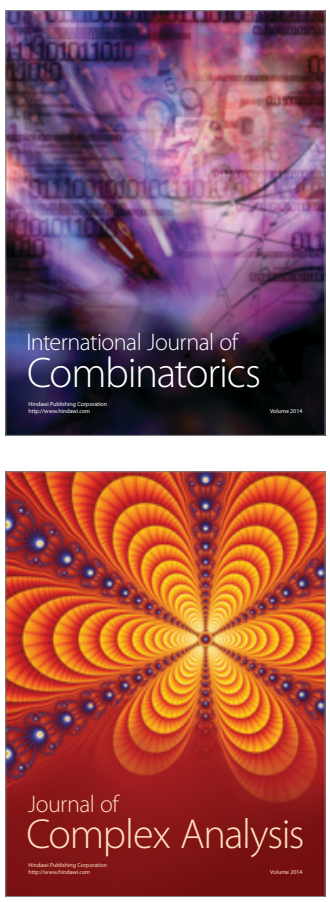

International Journal of

Mathematics and

Mathematical

Sciences
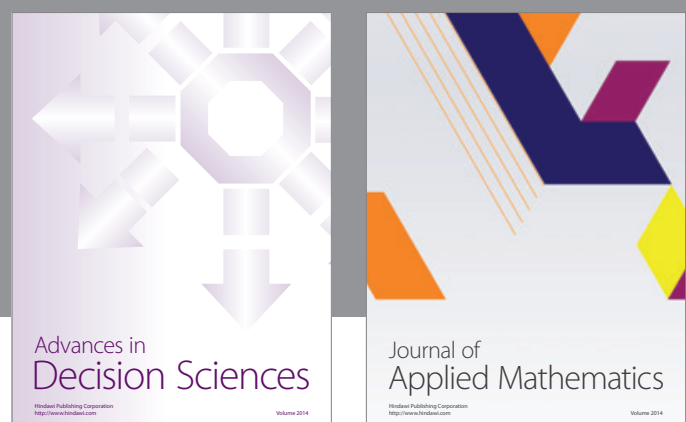

Journal of

Applied Mathematics
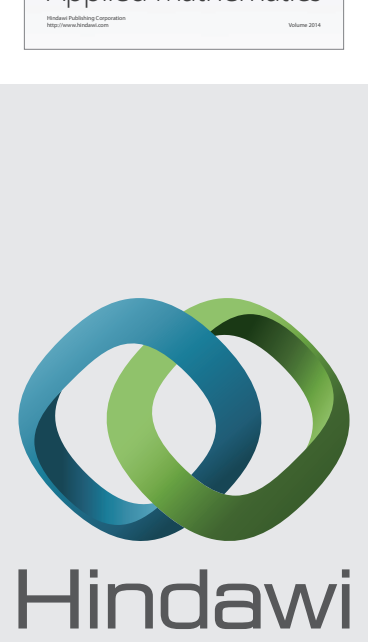

Submit your manuscripts at http://www.hindawi.com
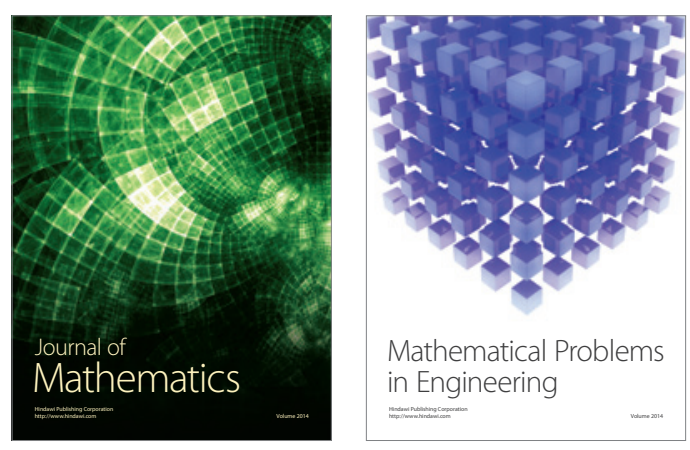

Mathematical Problems in Engineering
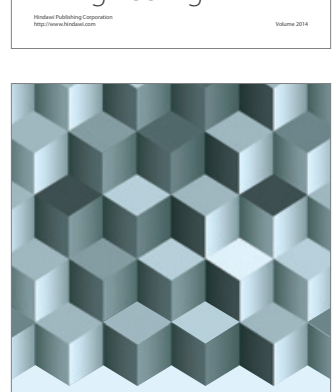

Journal of

Function Spaces
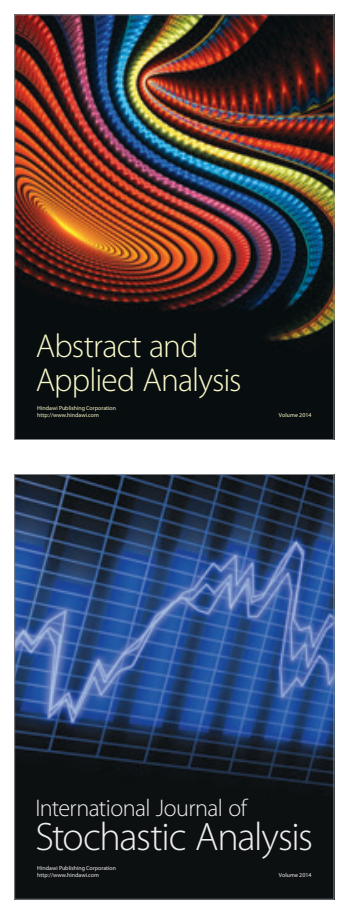

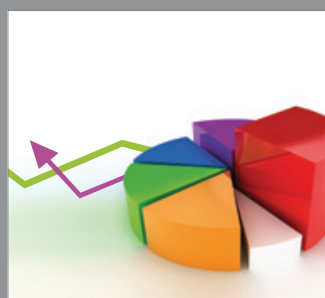

ournal of

Probability and Statistics

Promensencen
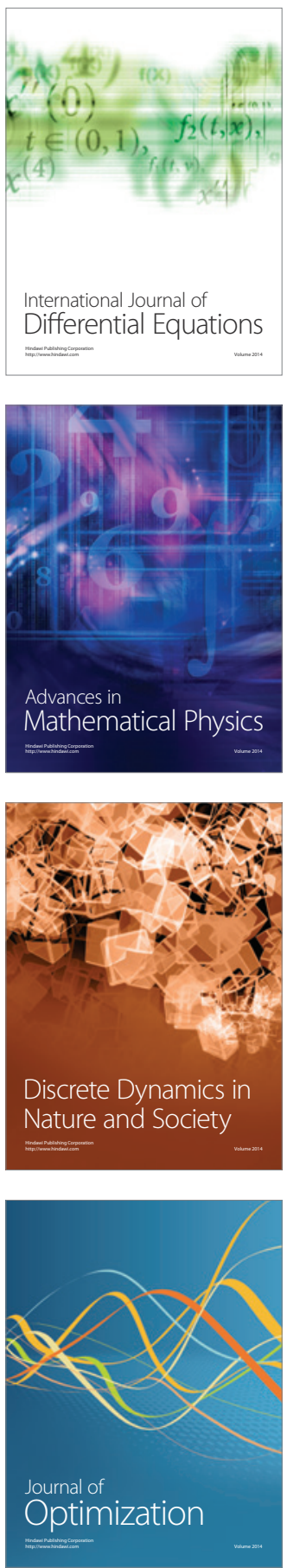